\title{
The Impact of Ankle Injuries on the Football Skills Performance of Amateur Teenaged Football Players
}

\author{
Li Li ${ }^{1, a, *}$ Jia Han ${ }^{2, b}$ and Nan Yang, ${ }^{3,4, c}$ \\ ${ }^{1}$ Yangpu District Teachers Training College, Shanghai, China \\ ${ }^{2}$ Kinesiology School, Shanghai University of Sport, Shanghai, China \\ ${ }^{3}$ Sport News and Media School, Shanghai University of Sport, Shanghai, China \\ ${ }^{4}$ Research Institute of Sport and Exercise, University of Cnaberra, Cnaberra, Austrlia \\ aLiLee_1973@126.com, bJia.Han@Canberra.edu.au, cyangnan@sus.edu.cn \\ *corresponding author
}

Keywords: Football, Sport Skills, Ankle, Sport Injuries, Correlation Analysis

\begin{abstract}
Backgrounds: Football players often get their ankles injured in the sport, which is believed to be one of the important factors that affect football players' sport career. However, to date, the effects of these injuries on football players' sports skills have not yet been thoroughly investigated. The present study is to examine the correlation between the ankle injury history and the performance of sports skills. Methods: A questionnaire survey on the history of sports injuries and football skills tests were conducted among 26 amateur football players from primary and secondary schools in Shanghai. Results: compared with healthy athletes, significant differences were identified between football players with and without ankle injury history in the success rate of shooting $(\mathrm{F} 1,24=5.769, \mathrm{p}=0.024)$. Conclusion: Positive correlation was found between different football skills. The ankle joint injury is significantly related to the shooting skills.
\end{abstract}

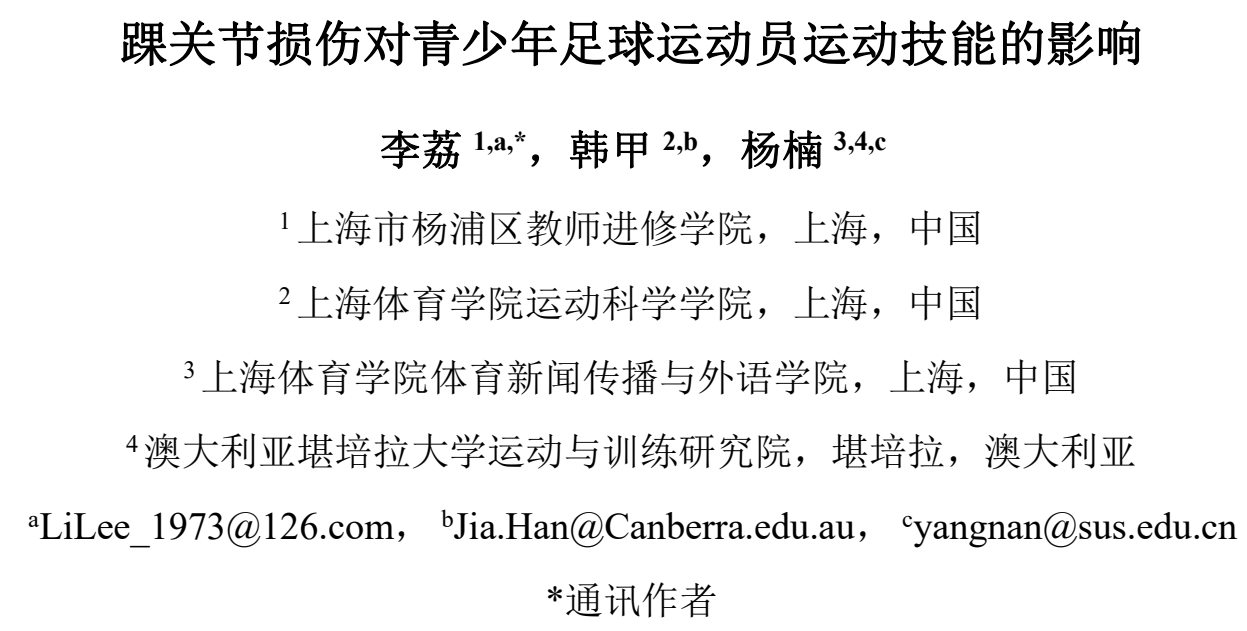

关键词：足球，运动技能，踝关节，运动损伤

摘要：研究背景：踝关节是足球运动员最容易发生运动损伤的关节之一，也是影响足球运动员 运动寿命的重要原因之一。但是这些损伤对足球运动员的运动技能的影响尚不明确。本研究旨在 
对于足球运动员的损伤史和运动技能表现进行相关性研究，以探究损伤对于运动技能的影响。研 究方法: 对来自上海市中小学的 26 名业余足球运动员运动损伤史进行问卷调查, 并进行足球相 关技能的测试。结果: 有损伤史的足球运动员与健康运动员相比，二者在射门成功率方面具有显 著性差异（ $F 1,24=5.769, p=0.024 ） 。$ 结论：踝关节的损伤对于足球运动员射门技能有显著影响， 足球运动技能的学习可以相互影响相互促进。

\section{1. 前言}

足球是一项极具鬼力的体育运动项目，具有高度的对抗性; 同时足球也是一项损伤高发的体 育项目，运动员受伤的几率非常高。足球运动员主要依靠脚和腿部来完成各项技术，因此足球活 动中腿部和脚部的运动损伤问题一直是运动员的一大困扰。在各种运动损伤中，踝关节是足球运 动员最容易发生运动损伤的关节之一，也是影响足球运动员运动寿命的重要原因之一[9]。因此， 青少年足球运动员在训练、比赛过程中必须重视踝关节损伤的预防。

踝关节即屈戊关节，是人体足部活动的主要关节。足球运动员的动作具有急停、急转、瞬间 万变等特点, 容易导致踝关节发生扭伤 [8]。踝关节属滑车形状的关节, 关节囊比较松弛, 外踝位 置低, 内踝位置高, 外侧韧带薄弱, 内侧韧带强韧, 足的内翻幅度大于外翻。从踝关节的这些解 剖特点可知踝关节是极为容易受伤的关节 $[8,11]$ 。

一般情况下，大多数伤者经过治疗都能痊愈，重返运动场，但是这些损伤对足球运动员运 动技能的发挥的影响尚不明确。基于此，本研究旨在通过对 26 名青少年业余足球运动员的足球 专项技能进行调查、评估和分析，探究踝关节损伤与运动员各项足球技能之间的具体关系。并针 对损伤康复后的青少年足球运动员的科学训练提出一些建议。

\section{2. 研究对象及方法}

\section{1 研究对象}

来自上海市中小学的业余足球运动员共 26 名（年龄： 13 至 16 岁, 平均年龄：14.5 岁）。运 动员平均训练年限 4.9 年。根据《滑铁卢惯用脚调查表（中文版）》[8]测试，所有受试运动员的 优势脚均为右脚。（见表 1)。

\section{2 受试者招募}

本研究的所有招募的受试者均在测试前进行问卷调查, 本研究的纳入标准包括并且满足要求 6 个月内无下肢受伤状况，无慢性疾病。由于测试人员均为未成年人，在获得监护人同意后，监 护人陪同下自愿签署知情同意书者，纳入到本次研究中。本研究的排除标准包括严重的下肢损 伤，下肢相邻关节骨折既往史，下肢手术史，骨骺发育不良，神经系统疾病，疾病急性期、肿瘤 等, 以上情况都将排除在本实验之外。

\section{3 测试方法}

\subsection{1 踝关节损伤问卷调查}

足球运动员踝关节的典型损伤主要有踝关节韧带软组织的损伤和踝部的骨折。[10]其中踝关节 的软组织损伤：轻微韧带损伤、不全韧带损伤及一条或多条韧带完全断裂为本研究的调查目标。 而踝部的骨折与下肢其它部位的骨折都被归为本研究的排除标准，因此招募的运动员中均无下肢 骨折历史。本研究共发放问卷 26 份, 收回 26 份, 回收率 $100 \%$ 。

\subsection{2 足球技能测试}


足球技能测试包括传球、盘带、射门，主要测试各项足球技能执行的成功率，精准度和完成时 间。测试场地和设备包括: 测量距离的皮尺，测量时间的秒表，足球若干，一面用于测量射门的 墙 $(7.33 \mathrm{~m} * 2.44 \mathrm{~m})$ ，室内人造草坪足球场，和 6 台摄像机。

测试准备阶段, 由教练带领受试者, 进行 20 分钟的热身活动, 热身活动包括慢跑、拉伸和球性 练习等。每位测试者分别在射门、传球和运球测试场地进行预测（预测 4-6 次）, 然后开始测 试, 测试在室内人造草坪的足球场进行, 气温 $20^{\circ} \mathrm{C}--25^{\circ} \mathrm{C}$, 要求每位测试者射门 8 次, 传球 28 次, 盘带 10 次。射门和传球测试起点有球释放装置, 左右随机。最终数据记录每项运动技能完 成的成功率、精准误和完成时, 其中成功率为成功次数与测试次数之比, 精准误表示足球偏离目 标的距离来表示, 即与精准度成反比。

(1) 射门测试

足球从足球释放装置中从受试者左侧或右侧随机、匀速释放出来, 当足球滚到指定区域

$(1.5 \mathrm{~m} * 1.5 \mathrm{~m})$, 受试者从起点以站立姿势慢跑至该指定区域踢球射门。测试者不可在踢球前停 球、控球。

目标球门为一面画在墙上的标准球门, 规格为 $7.33 \mathrm{mx} 2.44 \mathrm{~m}$ 。球门上有四个目标区域, 分别 是距离球门四个角横向 $1 \mathrm{~m}$,纵向 0.5 米的四个方形区域。射门共 8 次, 四个目标各两次。测量误 差的标准为击中点距离目标方形区域中心点的距离 $(\mathrm{cm}) 。$ 该项测试记录成功次数（击中目标 区域次数）、精准误及完成时间。

(2) 传球测试

足球从足球释放装置中从受试者左侧或右侧随机、匀速释放出来至指定区域 $(1.5 \mathrm{~m} * 1.5 \mathrm{~m})$, 受 试者从起点以站立姿势慢跑至该指定区域传球。此项要求受试者传球至 4 个目标区域中随机指定 的一个目标区域。受试者可自行选择 4 个目标区域之一进行传球。目标区域为 $2 \mathrm{~m} * 1 \mathrm{~m}$ 方框, 中 心有一个 $0.5 \mathrm{~m} * 0.25 \mathrm{~m}$ 的方形目标。 4 个目标区域与起点距离分别为 $4.2 \mathrm{~m}-7.9 \mathrm{~m}$ 。传球测试共 28 次 (每个目标 7 次)。该项测试记录测量距离误差、完成时间及成功击中目标区域次数。

(3) 盘带测试

受试者从起点 $1 \mathrm{~m}$ 处开始运球绕障碍物, 障碍物共有 7 个, 起终点距离为 $20 \mathrm{~m}$ 。每位受试者完 成 10 次，测试者绕过每个障碍物则被视为成功 1 次。该项测试记录受试者的成功次数、绕过 7 个障碍物的总时间以及绕过每个障碍物时的精度。

\section{4 统计学分析}

统计学分析使用 IBM SPSS 20.0 软件对运动足球技能成绩和踝关节扭伤历史进行 Pearson 相关 性检验和单因素方差分析，检验水平为 $\alpha=0.05$ 。

\section{3. 结果}

\section{1 受试者信息}

表 1：受试者基本信息（均值沶准差）

\begin{tabular}{cccccccc}
\hline $\begin{array}{c}\text { 受试 } \\
\text { 者 }\end{array}$ & 年齡 & 身高 & 体重 & \multicolumn{2}{c}{ 脚踝受伤史 } & \multicolumn{2}{c}{ 优势脚 } \\
$\mathrm{n}$ & & & & & & & \\
& & $\mathrm{cm}$ & $\mathrm{kg}$ & 有 & 无 & 左 & 右 \\
\hline 26 & $14.8 \pm 1.2$ & $172 \pm 7.0$ & $62.1 \pm 13.4$ & 12 & 14 & 0 & 26 \\
\hline
\end{tabular}

\section{2 损伤对足球运动技能的影响}


单因素方差分析结果表明, 有损伤史的受试者在射门成功率与未损伤受试者相比具有显著差异 $(\mathrm{F} 1,24=5.769, \mathrm{p}=0.024)$ 。

表 2: 踝关节损伤与足球运动技能的单因素方差分析

\begin{tabular}{cccc}
\hline & & $\mathrm{F}$ & $\mathrm{P}$ \\
\hline \multirow{3}{*}{ 射门 } & 项目 & 0.59 & $0.024^{*}$ \\
& 成功率 & 1.287 & 0.267 \\
& 精准误 & 0.114 & 0.739 \\
& 完成时 & 1.414 & 0.246 \\
传球 & 成功率 & 3.919 & 0.059 \\
& 精准误 & 0.111 & 0.742 \\
& 完成时 & 1.281 & 0.268 \\
盘带 & 成功率 & 0.619 & 0.439 \\
& 精准误 & 1.299 & 0.265 \\
\hline & 完成时 & &
\end{tabular}

成功率: 成功次数/测试次数;

* : $\mathrm{p}<0.05$, 具有显著性差异

精准误: 足球偏离目标的距离, 与精准度成反比;

完成时：完成各项测试的总耗时

\section{3 足球技能之间的相关性分析}

射门、盘带、传球的成功率之间显著相关 $(r=0.621,0.529,0.883 ; \mathrm{p}<0.01)$ 并且各项技能的 成功次数、精准误差、完成时间，三者之间也具有显著的相关性 $(\mathrm{p}<0.05)$ 。

\section{4. 讨论}

\section{1 足球技能}

足球技能测试是用于评判和预测足球运动员在训练和对抗比赛运动表现的主要评价手段, 是对 足球运动员认知、感知和运动表现综合能力的反馈 [1], 因此足球技能测试除了测试本身要求较 好的内在效度以外, 还需要具备较高的外在效度。在以往的研究中, 结合足球比赛的自身特点, 一直将传球、盘带、射门作为足球技能测试的主要项目。一项对 1996-2007 年间发表的有关足球 技能测试进行系统性回顾的研究指出指出, 传球、盘带和射门的足球运动技能的测试更多的侧重 于足球技巧的测试而非足球技能, 而且由于缺乏统一的测试规范, 脱离真实的训练和比赛环境, 技能测试结果存在“天花板效应”。[2] 2010 年 Russell [6] 等学者对传统的传球、盘带和射门技能 测试进行了改良, 结合摄像技术从成功率、精准度和完成时三项指标不同层面分别评价每项技 能, 实验证明这种测试方法具有较高的内在效度和外在效度。本研究采用了 Russell 的足球技能 测试方法 [6], 通过相关性分析发现传球、盘带、射门三者的技能测试之间具有显著的相关性, , 结合运动技能学习的正迁移效应[7], 说明各项足球技能的学习和掌握是相互促进的关系。此 外, 在足球技能的各项指标之间, 传球技能的成功率与完成时呈显著正相关, 即成功率与完成速 度呈负相关; 盘带和射门技能测试的精准误和完成时呈显著的正相关, 其中以盘球的精准度与完 成时间的相关性最为显著 $(\mathrm{r}=0.753, \mathrm{p}=0.00)$ 。因为精准误代表足球偏离目标的距离, 与精准度 成反比, 即完成速度亦与精准度呈负相关, 也就是说速度越快, 动作的精准度就会下降。

\section{2 踝关节损伤对足球技能的影响}


通过单因素方差分析, 结果显示有踝关节损伤史的足球运动员射门成功率显著低于未损伤运动 员。这一发现与以往的研究结果一致, 踝关节损伤后显著影响足球运动员的运动表现 [3]。本研 究在对有无损伤史与运动技能的单因素方差分析中, 发现有损伤史运动员射门技能受到显著影 响, 而在盘带和传球技能的比较中差异并不显著。此外, 踝关节的损伤会显著影响关节的活动能 力, 亦有研究指出, 射门时, 踝关节需要较大的垂直方向的运动和较小的切入角度才能拉出理想 的曲线球[4]。因此对于足球运动员来说, 踝关节的损伤将会显著影响运动员的运动技能发挥。

\section{3 研究的不足与展望}

本实验是基于横断面的相关性研究, 足球技能与运动损伤的相关性有赖于更加严密的随机对照 实验验以证其因果关系; 今后的研究方向可以探究不同损伤类型对于足球专项技能的影响, 这将 有利于足球运动员、足球教练和医疗团队进行精准化的训练、体育教学和损伤预防, 以及为已损 伤的运动员提供科学、针对性的康复锻炼指导。

\section{5. 结论与建议}

本研究通过对 27 名青少年业余足球运动员的踝关节运动损伤史和他们的足球技能的调查、测 试和分析发现各项足球运动技能的学习可以相互影响相互促进, 各项运动技能完成的速度与准确 性相互制约; 踝关节的扭伤对于足球运动员射门技能的影响较大。

踝关节扭伤是其中较为严重的伤害之一, 严重影响运动员的训练和比赛, 制约着运动队训练水 平的提升和比赛成绩的提高, 应该加以重视和预防。无论训练还是比赛, 足球教练员和队员应在 思想上予以高度重视, 改善训练和比赛的环境, 加强身体素质的训练, 提高下肢肌肉及踝关节力 量尽量减少踝关节损伤的发生。

\section{参考文献}

[1] Ali, A., Measuring soccer skill performance: A review[J]. Scandinavian Journal of Medicine andScience in Sports, 2011. 21(2): p. 170-183.

[2] Ali, A., et al., Reliability and validity of two tests of soccer skill[J]. Journal of Sports Sciences, 2007. 25(13): p. 1461-1470.

[3] Han, J., et al., Level of competitive success achieved by elite athletes and multi-joint proprioceptive ability[J]. J Sci Med Sport, 2015. 18(1): p. 77-81.

[4] Hong, S., et al., Characteristics of Ball Impact on Curve Shot in Soccer[J]. Procedia Engineering, 2013. 60: p. 249-254.

[5] Nan Y, Waddington G, Adams R, et al. Translation, cultural adaption, and test-retest reliability of Chinese versions of the Edinburgh Handedness Inventory and Waterloo Footedness Questionnaire[J]. Laterality, 2018, 23(3):255.

[6] Russell, M., D. Benton, and M. Kingsley, Reliability and construct validity of soccer skills tests that measure passing, shooting, and dribbling[J]. J Sports Sci, 2010. 28(13): p. 1399-408.

[7] 曹跃兴, 对运动技能迁移的研究[J]. 体育科技, 2004(01): p. 19-21.

[8] 杜邦胜. 足球运动员踝关节损伤的预防与处理 [J]. 体育科技文献通报, 2006, 14(9):52-52.

[9] 高静. 足球运动员踝关节损伤的原因及康复研究 [J]. 当代体育科技, 2013, 3(33):22-22.

[10] 陆勇, 贾文. 高校足球运动常见运动损伤的相关因素调查 [J]. 上海体育学院学报, 2003, 27(6):70-70.

[11] 张扬, 王太林. 业余足球运动员踝关节损伤的原因及治疗 [J]. 体育科技, 2011, 32(4):65-67. 